\title{
Avaliação das Estimativas de Precipitação do Produto 3B43-TRMM do Estado do Amazonas
}

\author{
Catherine Torres de Almeida ${ }^{1}$, Rafael Coll Delgado ${ }^{1}$, José Francisco de Oliveira Junior ${ }^{1}$, \\ Givanildo Gois ${ }^{1}$, Alessandro Sarmento Cavalcanti ${ }^{2}$
}

${ }^{1}$ Departamento de Ciências Ambientais, Universidade Federal Rural do Rio de Janeiro - UFRRJ, Seropédica/RJ, Brasil ${ }^{2}$ Departamento de Engenharia Elétrica, Centro de Estudos Superiores de Maceió - CESMAC, Maceió/AL, Brasil

\begin{abstract}
RESUMO
Para avaliar os dados de precipitação pluvial via satélite no estado do Amazonas, compararam-se as estimativas do produto 3B43 do satélite TRMM (2004-2008) com dados de sete Estações Meteorológicas Convencionais (EMC). A comparação foi baseada nos seguintes parâmetros estatísticos: Erro Médio (EM), Raiz do Erro Médio Quadrático (REMQ), coeficiente de correlação linear (r) e índice de concordância de Wilmott (d). As estimativas do TRMM foram similares aos dados de superfície e representaram bem a variabilidade sazonal das chuvas. Os dados apresentaram alta correlação linear $(r=0,83)$, alto índice de concordância $(d=0,85)$ e REMQ satisfatório (66,6 mm/mês). Dessa forma, as estimativas de precipitação pluvial do produto 3B43 podem ser utilizadas como uma fonte alternativa de dados de qualidade.
\end{abstract}

Palavras-chave: Amazônia, chuva, sensoriamento remoto, validação.

\section{Assessment of Rainfall Estimates from the TRMM-3B43 Product in the State of Amazonas}

\begin{abstract}
The aim of this study was to evaluate the rainfall data via satellites in Amazonas state, Brazil. To this end, the estimates from the TRMM-3B43 product (2004-2008) were compared with data from seven Conventional Weather Stations (CWS). The comparison was based on the following statistical parameters: Average Error (AE), Root Mean Square Error (RMSE), linear correlation coefficient ( $r$ ), and Wilmott's index of agreement (d). The TRMM-3B43 estimates were similar to the surface data and represent well the seasonal variability of rainfall. The data showed high linear correlation $(r=0.83)$, high index of agreement $(d=0.85)$, and satisfactory RMSE ( $66.6 \mathrm{~mm} / \mathrm{month})$. Therefore, rainfall estimates from the TRMM-3B43 product can be used as an alternative source of quality data.
\end{abstract}

Keywords: Amazon, rainfall, remote sensing, validation. 


\section{INTRODUÇÃO}

A precipitação pluvial é uma das principais características determinantes do clima amazônico (Fisch et al., 1998), sendo responsável pela distinção entre as estações chuvosas e secas. A Bacia Amazônica é um dos principais centros de atividades convectivas na região tropical do planeta, com uma área de cinco milhões de quilômetros quadrados (Ferreira da Costa et al., 1998). Dessa forma, é de extrema importância entender a dinâmica pluviométrica desta região, bem como os mecanismos que organizam e promovem a precipitação e sua relação com o funcionamento da floresta (Molion, 1987; Marengo et al., 2008).

Também faz-se necessário, diante da crescente preocupação com as mudanças climáticas, monitorar a variabilidade das chuvas em relação à modificação antrópica da paisagem amazônica e ao aumento na temperatura global, pois seus efeitos podem ter sérias consequências para o ecossistema amazônico (Adams et al., 2009). Segundo Marengo \& Betts (2011), o desmatamento e as mudanças climáticas podem prejudicar severamente o funcionamento da Amazônia como ecossistema florestal, resultando na perda de biodiversidade, redução de sua capacidade de reter carbono, enfraquecimento do ciclo hidrológico regional, aumento da temperatura do solo e, eventualmente, impelindo a Amazônia a um processo gradual de savanização.

O monitoramento pluviométrico de uma região requer uma grande densidade de pluviômetros ou telepluviômetros bem distribuídos espacialmente, uma vez que a escala de medição destes equipamentos é pontual (Kidder \& Haar, 1995), não fornecendo valores representativos para grandes áreas. Por possuir grande extensão territorial e difícil acesso em densas áreas de floresta, a região Amazônica apresenta baixa densidade de estações meteorológicas convencionais e automáticas, o que pode causar incertezas significativas no entendimento dos diferentes processos relativos aos mecanismos dinâmicos do clima e sua variabilidade espacial (Nobre et al., 2009). Além disso, a grande quantidade de falhas existentes nos dados dessas estações meteorológicas também prejudica uma análise temporal da variabilidade climática da região.

Devido ao elevado custo e à dificuldade na obtenção de medidas em áreas de difícil acesso, simulações numéricas e dados de satélite têm sido utilizados para estudos climatológicos e hidrológicos (Longo et al., 2004; Adams et al., 2009). Os dados provindos de satélites são uma alternativa para suprir as deficiências dos dados de superfície na estimativa da precipitação pluvial, pois estes são fornecidos em alta densidade espacial e temporal (Oliveira et al., 2014). O satélite TRMM (Tropical Rainfall Measuring Mission) foi lançado em 1997 pela NASA (National Aeronautics and Space Agency) e pela JAXA (National Space Development Agency of Japan) com o objetivo de monitorar a precipitação sobre os trópicos (Kummerow et al., 2000). Para garantir que esse satélite forneça estimativas consistentes é necessário validar essas informações com as medidas de superfície, visando quantificar sua precisão e exatidão.

Nesse contexto, o objetivo deste trabalho é comparar as estimativas de precipitação pluvial do satélite TRMM (produto 3B43) com dados pluviométricos de superfície provenientes de estações meteorológicas convencionais no estado do Amazonas, no período de 2004 a 2008.

\section{MATERIAL E MÉTODOS}

\subsection{Caracterização da área de estudo}

O Amazonas é o maior estado brasileiro, com uma área de 1.559.159,148 km² e uma população de 3.483.985 habitantes (IBGE, 2012). Ao todo possui 62 municípios e a capital Manaus é a cidade mais populosa da região Norte e o sétimo município mais populoso do Brasil (IBGE, 2011). O estado do Amazonas possui a maior área de floresta conservada no Brasil, uma vez que $98 \%$ de sua área ainda mantém sua cobertura de floresta tropical (FAS, 2014). Cerca de 50\% de sua área é protegida por unidades de conservação, tais como reservas extrativistas, parques nacionais, estaduais e federais, e também terras indígenas (Amazonas, 2009).

De acordo com a classificação de Köppen, a região Amazônica apresenta o clima do tipo "A", com três subclimas: o equatorial chuvoso ("Af"), o tropical de monção ("Am") e o tropical seco e úmido ("Aw"), sendo que o tropical de monção abrange a maior parte da região. Em escalas de tempo sazonais, a temperatura média do ar não apresenta muita variação, o que se deve aos altos valores de radiação solar incidente ao longo do ano. Os valores da temperatura média do ar estão entre $24^{\circ} \mathrm{C}$ e $26^{\circ} \mathrm{C}$ com amplitude anual de 
$1{ }^{\circ} \mathrm{C}$ a $2{ }^{\circ} \mathrm{C}$. A precipitação média da região Amazônica é de aproximadamente $2.300 \mathrm{~mm}$. ano ${ }^{-1}$. A estação chuvosa é compreendida entre novembro e março, e a estação seca, entre maio e setembro. Os meses de abril e outubro são meses de transição entre um regime e outro (Figueroa \& Nobre, 1990).

\subsection{Dados de superfície e de satélite}

Os dados diários de precipitação pluvial foram obtidos do Banco de Dados Meteorológicos para Ensino e Pesquisa (BDMEP) do INMET (Instituto Nacional de Meteorologia) (INMET, 2012), referentes a sete Estações Meteorológicas Convencionais (EMC) no estado do Amazonas, localizadas nos seguintes municípios: Barcelos, Codajás, Eirunepé, Fonte Boa, Manaus, Manicoré e São Gabriel da Cachoeira. Essas estações foram escolhidas devido à qualidade dos dados para o período de 2004 a 2008, com porcentagem de falhas inferior a 3\%. Os dados de precipitação pluvial diários foram convertidos em dados acumulados mensais. Estes somente foram calculados para os meses que possuíam ao menos $90 \%$ dos dados de precipitação.

Os dados de precipitação pluvial do satélite TRMM produto 3B43 foram obtidos no formato NetCDF (TRMM, 2014). Esse produto fornece dados com resolução espacial de aproximadamente $30 \mathrm{~km}$ e resolução temporal mensal. Utilizou-se o programa ArcGIS versão $10.2^{\circledR}$ para a conversão dos dados, originalmente em mm.h ${ }^{-1}$ para mm.mês ${ }^{-1}$, e para a seleção dos pontos TRMM mais próximos das EMCs, cujos dados foram utilizados para a comparação com os provindos das EMCs, para os anos de 2004 a 2008. No caso da EMC de São Gabriel da Cachoeira, consideraram-se dois pontos TRMM, devido à distância similar entre estes e a EMC. Foi calculada a média aritmética da precipitação mensal dos dois pontos TRMM como medida representativa dessa localidade. A localização das EMCs e dos pontos selecionados do TRMM encontra-se na Figura 1.

\subsection{Validação das estimativas do satélite}

Para a comparação da precipitação pluvial estimada pelo produto 3B43 do TRMM, em relação aos valores obtidos em superfície pelas EMCs, foram utilizados os seguintes parâmetros estatísticos: Erro Médio (EM, Equação 1), Raiz do Erro Médio Quadrático (REMQ, Equação 2), coeficiente de correlação linear de Pearson (r, Equação 3) e índice de concordância (d, Equação 4) proposto por Willmott et al. (1985). As equações utilizadas são apresentadas abaixo:

$\mathrm{EM}=\frac{\sum_{\mathrm{i}=1}^{\mathrm{n}}\left(\mathrm{P}_{\mathrm{i}}-O_{i}\right)}{\mathrm{n}}$

$R E M Q=\sqrt{\frac{\sum_{i=1}^{n}\left(P_{i}-O_{i}\right)^{2}}{n}}$

$r=\frac{\sum_{i=1}^{n}\left(P_{i}-\bar{P}\right) *\left(O_{i}-\bar{O}\right)}{\sqrt{\sum_{i=1}^{n}\left(P_{i}-\bar{P}\right)^{2} * \sum_{i=1}^{n}\left(O_{i}-\bar{O}\right)^{2}}}$

$d=1-\left[\frac{\sum_{i=1}^{n}\left(P_{i}-O_{i}\right)^{2}}{\sum_{i=1}^{n}\left(\left|P_{i}-O_{i}\right|+\left|O_{i}-\bar{O}\right|\right)^{2}}\right]$

em que: $\mathrm{P}_{\mathrm{i}}=$ precipitação estimada $(\mathrm{mm})$ pelo satélite TRMM no intervalo de tempo $\mathrm{i} ; \mathrm{O}_{\mathrm{i}}=$ precipitação observada ( $\mathrm{mm}$ ) em determinada EMC no intervalo de tempo i; $\mathrm{n}=$ número de dados analisados; $\bar{P}=$ valor médio estimado de precipitação $(\mathrm{mm})$ pelo satélite TRMM; $\bar{O}=$ valor médio observado de precipitação $(\mathrm{mm})$ em determinada EMC.

O coeficiente de correlação foi avaliado pelo teste t-Student, ao nível de significância de 5\%. Utilizou-se o software R, versão 3.1.0 (R Development Core Team, 2014) para a realização das análises estatísticas do conjunto de dados no período de 2004-2008.

\section{RESULTADOS E DISCUSSÃO}

A distribuição temporal da precipitação pluvial mensal no período de 2004 a 2008 para ambas as fontes de dados (TRMM e EMC) nas sete localidades avaliadas (Figura 2) mostrou que as estimativas do satélite TRMM apresentaram similaridade aos dados obtidos das EMCs. O produto 3B43 também estimou corretamente a variabilidade sazonal da precipitação, o que permite a utilização desses dados para a identificação das estações secas e chuvosas no estado do Amazonas.

Os resultados também mostraram que os dados estimados pelo produto 3B43 do TRMM e observados nas EMCs apresentaram alta e significativa correlação em todos os municípios avaliados (Figura 3a-g), com o coeficiente $\mathrm{r}$ de 0,83 (intervalo de confiança a $95 \%$ 


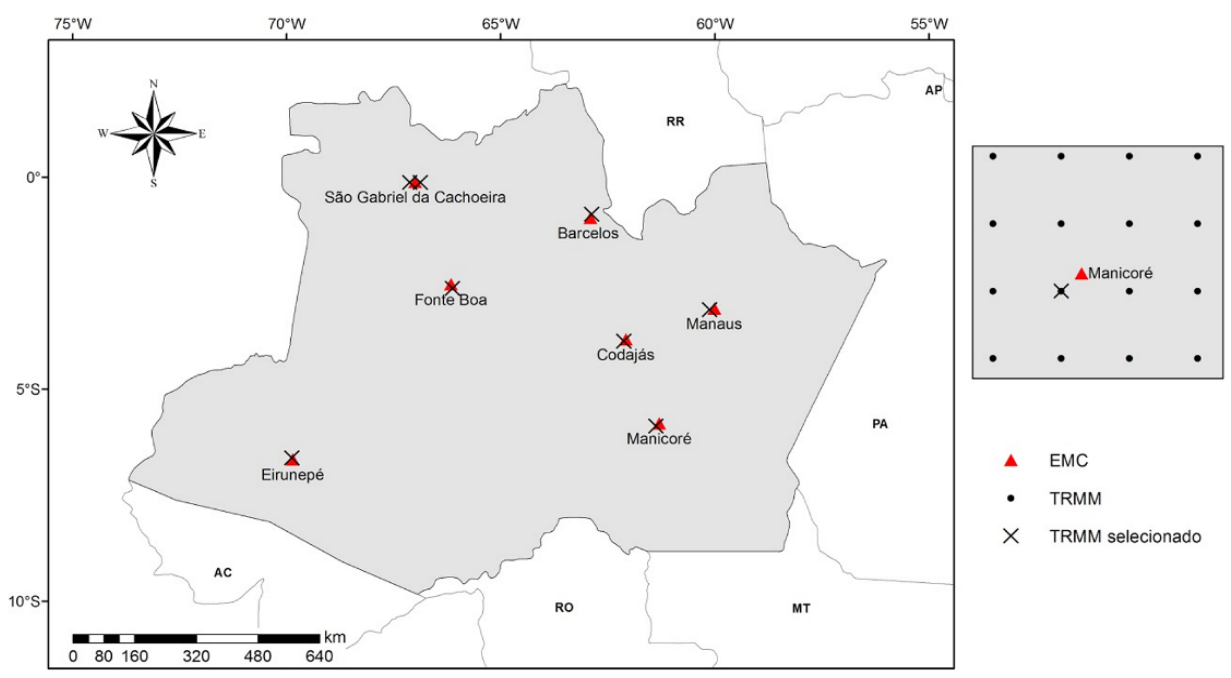

Figura 1. Localização das EMCs e dos pontos do TRMM mais próximos a elas.

Figure 1. Location of CWS and the TRMM points closest to them.
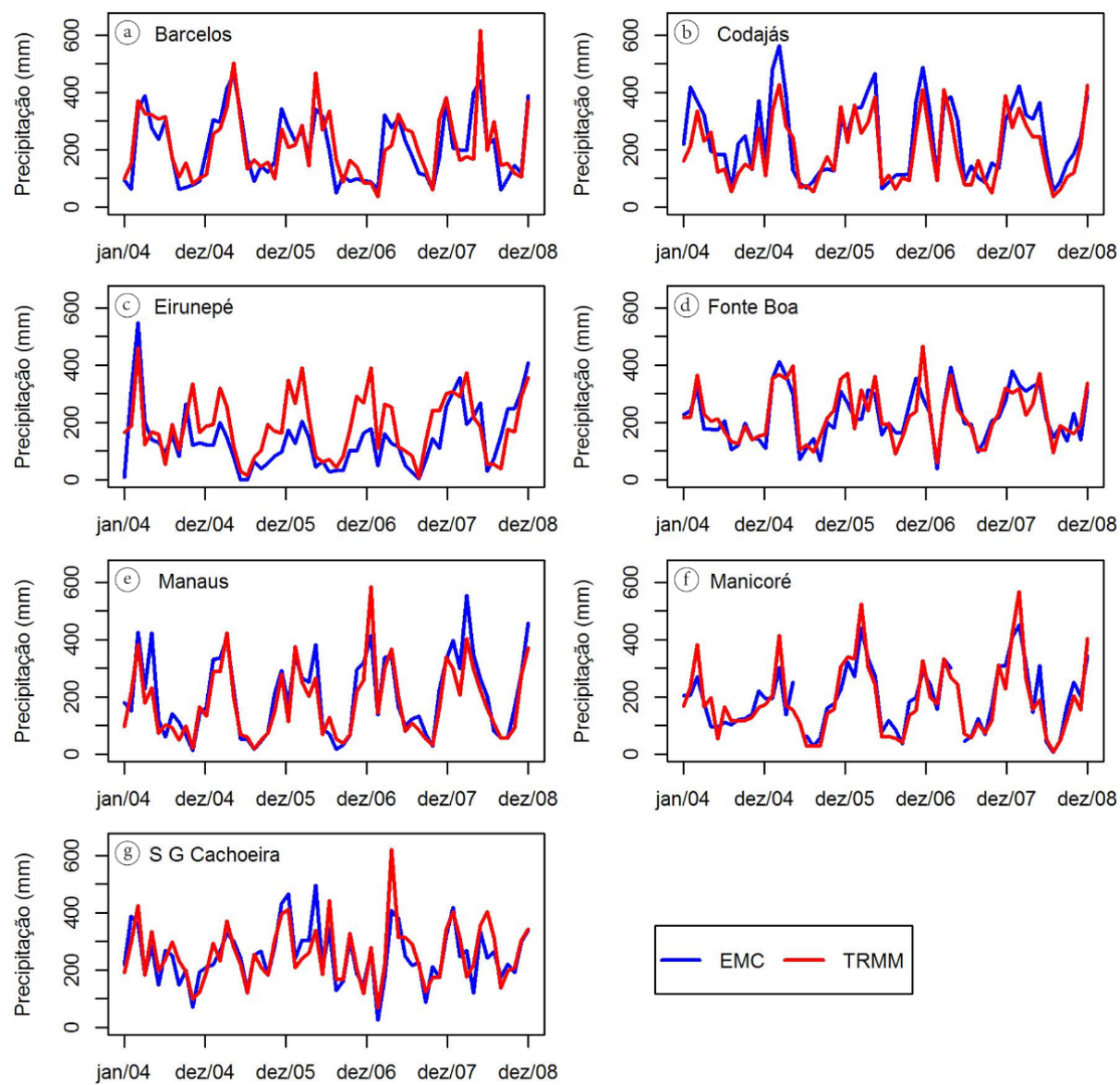

Figura 2. Precipitação pluvial mensal (mm) de janeiro de 2004 a dezembro de 2008: comparação entre TRMM e EMCs nos sete municípios avaliados.

Figure 2. Monthly rainfall (mm) from January 2004 to December 2008: comparison between TRMM and CWS in the seven municipalities evaluated. 
de probabilidade de 0,80 a 0,86 ), significativo a $5 \%$ de significância (p-valor <2,2e-16), considerando os dados de todas as localidades conjuntamente (Figura $3 \mathrm{~h}$ ). O índice d também apresentou altos valores (próximos de 1) para todas as EMCs (Figura 3).

Os municípios Manicoré, Fonte Boa e Manaus apresentaram os menores valores de REMQ (49,7 mm,
$52,8 \mathrm{~mm}$ e $58,9 \mathrm{~mm}$, respectivamente), com grande proximidade entre os dados do TRMM e os dados de superfície (Figuras 2e, 2d e 2f). Os municípios Manaus e Manicoré também apresentaram os maiores valores de coeficiente de correlação e índice de concordância que, juntamente com os baixos valores de REMQ,
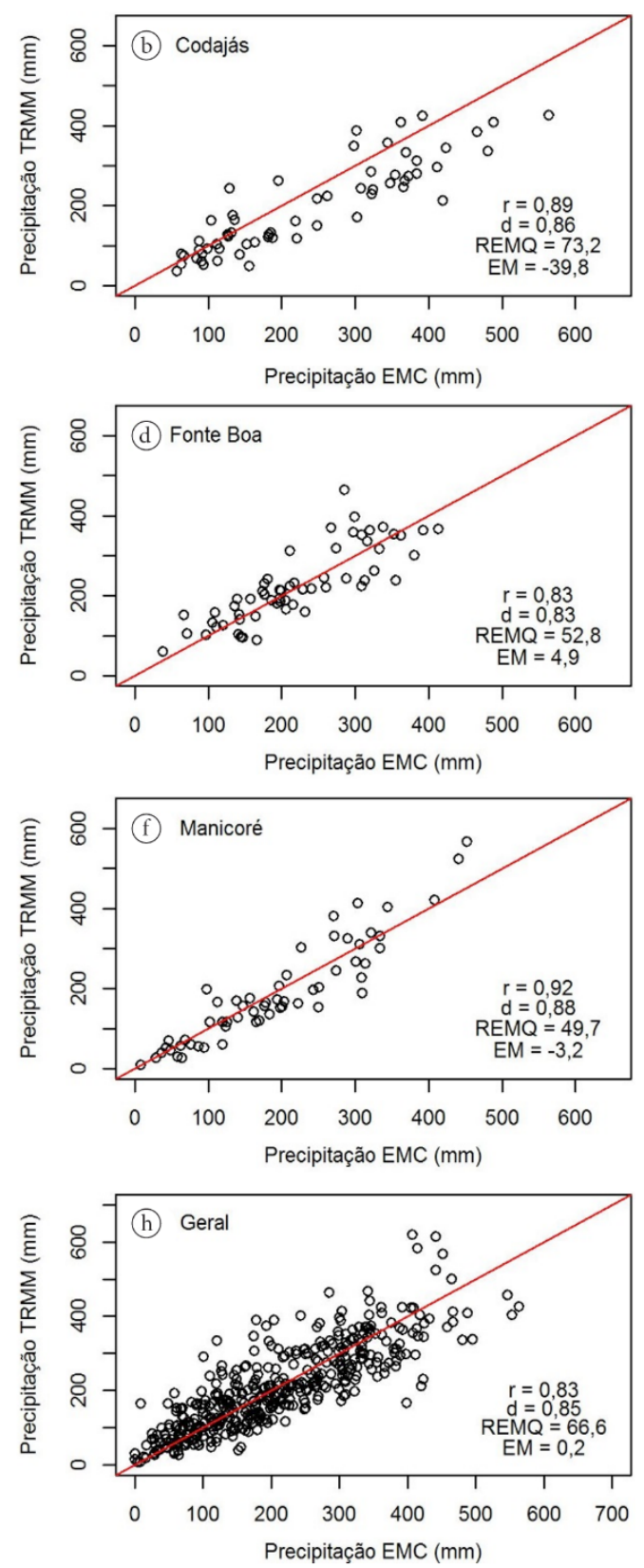

Figura 3. Diagrama de dispersão individual para cada localidade estudada (a-g) e geral, considerando todos os dados conjuntamente $(\mathrm{h})$.

Figure 3. Scatter diagram for the seven locations studied (a-g) and considering all the data together (h). 
indicam que nessas localidades os dados do TRMM 3B43 apresentam boa precisão e exatidão.

Além disso, Manaus, Manicoré e Codajás, municípios localizados mais ao leste do estado, apresentaram erro médio negativo (Figuras 3b, 3e e 3f), indicando maior tendência do TRMM de subestimar a precipitação mensal nessas localidades. As demais localidades apresentaram EM positivo, mostrando que o TRMM apresentou maior tendência de superestimar a precipitação.

A variabilidade da precipitação pluvial na região Amazônica se deve à atuação de sistemas meteorológicos sinóticos (Zona de Convergência Intertropical do Atlântico - ZCIT, Alta da Bolívia - AB, Vórtices Ciclônicos de Altos Níveis - VCAN e Zona de Convergência do Atlântico Sul - ZCAS) e de mesoescala (Friagens, Cumulunimbus Isolados e Linhas de Instabilidade - LI), seguido dos modos de variabilidade climática (El Niño, Oscilação Sul - ENOS e Anomalias das Temperaturas da Superfície do Mar - TSM dos Oceanos Pacífico Equatorial e Atlântico), que influenciam os padrões de chuvas na região (Ananias et al., 2010; Reboita et al., 2010). Variações espaciais desses sistemas meteorológicos sob o estado do Amazonas, bem como possíveis erros nos registros das EMCs, podem influenciar na super ou subestimação do TRMM.

Eirunepé foi a estação que apresentou maior valor de erro médio e de REMQ, pois os dados do TRMM foram superiores aos dados de superfície para a maioria dos meses nos anos 2005, 2006 e 2007
(Figura 2c), que foram anos de baixa precipitação de acordo com os valores registrados pela EMC. Eirunepé também apresentou os menores valores de coeficiente de correlação $(r=0,71)$ e índice de concordância $(d=0,71)$ (Figura $3 c$ ). O pior desempenho desta estação em relação às demais pode estar relacionado à sua localização mais ao sul do estado, sob influência de diversos sistemas meteorológicos, bem como pela sua menor pluviosidade, sendo mais influenciada pela maior abrangência do satélite. A baixa intensidade das chuvas no ano de 2005 em Eirunepé pode ser explicada pelo aquecimento anômalo do Atlântico norte tropical, que resultou em um período de forte seca na região sudoeste da Amazônia (Marengo et al., 2008).

Estudos da variabilidade interanual da precipitação na Amazônia também relacionam eventos de seca e de aumento no regime pluviométrico ao modo de variabilidade climática El Niño-Oscilação Sul (ENOS), devido a anomalias da Temperatura da Superfície do Mar (TSM) no Oceano Pacífico (Andreoli et al., 2012; Araújo et al., 2013). Considerando a média anual geral da precipitação total (Figura 4a), observou-se que o ano de 2008 apresentou maior média de chuva em comparação aos demais anos da série. Essa maior ocorrência coincidiu com a fase positiva do ENOS (La Niña) que, segundo o CPTEC (2014), teve a intensidade categorizada como forte neste ano. Decorrente dessa maior precipitação, o EM neste ano (Figura 4b) foi elevado, resultando na subestimativa
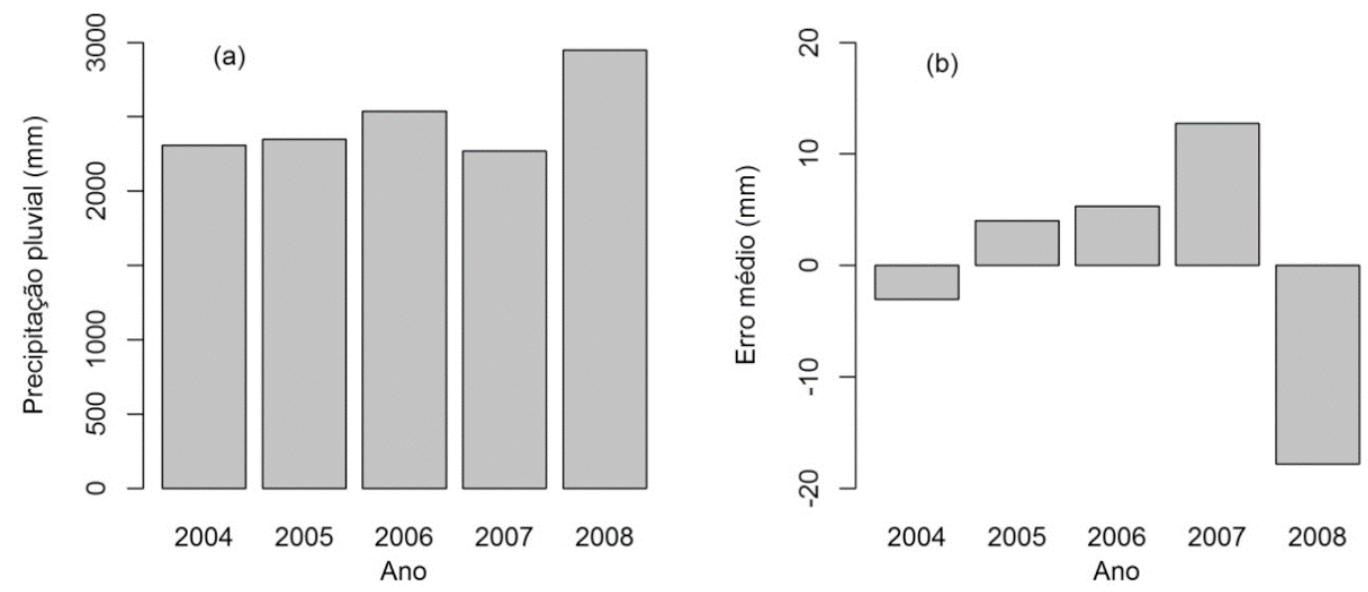

Figura 4. Média da precipitação pluvial total anual ( $\mathrm{mm})$ e do erro anual (mm) para todas as EMCs. Figure 4. Average of annual total rainfall $(\mathrm{mm})$ and annual error $(\mathrm{mm})$ for all CWS. 
da precipitação pluvial pelo TRMM. Vila et al. (2009) também observaram casos de subestimativa de chuvas mais intensas. Esses autores utilizaram o produto TRMM 3B42RT (dados de precipitação pluvial a cada 3 horas), combinando os dados de satélite com dados de superfície para detectar chuvas na América do Sul.

Assim como observado por outros autores (Collischonn et al., 2007; Collischonn et al., 2008; Nóbrega et al., 2008, Karaseva et al., 2012; Oliveira et al., 2014), os resultados indicam que as estimativas de precipitação pluvial fornecidas pelo TRMM são consistentes, porém apresentam alguns erros. As diferenças existentes entre os dados derivados do satélite TRMM e obtidos pelas estações meteorológicas podem ser uma consequência da diferença de escala entre eles, pois o pluviômetro é uma estimativa pontual, enquanto que o satélite representa uma estimativa média no pixel.

\section{CONCLUSÃO}

As estimativas de precipitação pluvial do produto 3B43 do satélite TRMM são uma boa fonte alternativa de dados para a região Amazônica segundo os parâmetros estatísticos, representando bem a variabilidade sazonal da precipitação pluvial no período de estudo. Dessa forma, esse produto pode auxiliar em estudos pluviométricos de regiões com baixa densidade de informações de superfície, assim como no preenchimento de falhas e homogeneização dos dados de precipitação em estações meteorológicas convencionais.

\section{AGRADECIMENTOS}

Ao INMET e à NASA pela disponibilização dos dados de precipitação. À CAPES, pela concessão de Bolsa de Mestrado à primeira autora e ao CNPq pelo financiamento do projeto de pesquisa relacionado a este trabalho.

\section{STATUS DA SUBMISSÃO}

Recebido: 11 set., 2014

Aceito: 30 mar., 2015
AUTOR(ES) PARA CORRESPONDÊNCIA:

\section{Catherine Torres de Almeida}

Departamento de Ciências Ambientais, Universidade Federal Rural do Rio de Janeiro UFRRJ, CEP 23851-970, Seropédica, RJ, Brasil e-mail: cathe.torres@gmail.com

\section{REFERÊNCIAS}

Adams DK, Souza EP, Costa AA. Convecção úmida na Amazônia: implicações para modelagem numérica. Revista Brasileira de Meteorologia 2009; 24(2): 168-178. http:// dx.doi.org/10.1590/S0102-77862009000200006.

Amazonas. Governo do Estado. A Floresta Amazônica e seu papel nas mudanças climáticas. Manaus: SDS:CECLIMA; 2009.

Ananias DS, Souza EB, Souza PFS, Souza AML, Vitorino MI, Teixeira GM et al. Climatologia da estrutura vertical da atmosfera em novembro para Belém-PA. Revista Brasileira de Meteorologia 2010; 25(2): 218-226. http:// dx.doi.org/10.1590/S0102-77862010000200006.

Andreoli RV, Ferreira de Souza RAF, Kayano MT, Candido LA. Seasonal anomalous rainfall in the central and eastern Amazon and associated anomalous oceanic and atmospheric patterns. International Journal of Climatology 2012; 32(8): 1193-1205. http://dx.doi.org/10.1002/joc.2345.

Araújo RG, Andreoli RV, Candido LA, Kayano MT, Souza RAF. A influência do evento El Niño: oscilação sul e atlântico equatorial na precipitação sobre as regiões norte e nordeste da América do Sul. Acta Amazonica 2013; 43(4): 469-480. http://dx.doi.org/10.1590/S0044-59672013000400009.

Centro de Previsão de Tempo e Estudos Climáticos CPTEC. El Niño e La Niña [online]. [citado 2014 jun. 21]. Disponível em: http://enos.cptec.inpe.br/

Collischonn B, Allasia D, Collischonn W, Tucci CEM. Desempenho do satélite TRMM na estimativa de precipitação sobre a bacia do Paraguai superior. Revista Brasileira de Cartografia 2007; 59(1): 93-99.

Collischonn B, Collischonn W, Tucci CEM. Daily hydrological modeling in the Amazon basin using TRMM rainfall estimates. Journal of Hydrology 2008; 360(1-4): 207-216. http://dx.doi.org/10.1016/j.jhydrol.2008.07.032.

Ferreira da Costa R, Feitosa JRP, Fisch G, Souza SS, Nobre CA. Variabilidade diária da precipitação em regiões de floresta e pastagem na Amazônia. Acta Amazonica 1998; 28(4): 395-408.

Figueroa SN, Nobre CA. Precipitions distribution over central and western tropical south America. Climanálise Boletim de Monitoramento e Análise Climática 1990; 5(6): 36-45.

Fisch G, Marengo JA, Nobre CA. Uma revisão geral sobre o clima da Amazônia. Acta Amazonica 1998; 28(2): 101-126. 
Fundação Amazonas Sustentável - FAS. Relatório de gestão 2011 [online]. [citado 2014 fev. 18]. Disponível em: http://fas-amazonas.org/versao/2012/wordpress/ wp-content/uploads/2012/06/RELAT\%C3\%93RIO-DEGEST\%C3\%83O-2011-FAS.pdf

Instituto Brasileiro de Geografia e Estatística - IBGE. Banco de dados agregados [online]. [citado 2012 out. 22]. Disponível em: http://www.sidra.ibge.gov.br

Instituto Brasileiro de Geografia e Estatística - IBGE. Sinopse do censo demográfico 2010. Rio de Janeiro: IBGE; 2011.

Instituto Nacional de Meteorologia - INMET. Banco de dados meteorológicos para ensino e pesquisa [online]. [citado 2012 out. 22]. Disponível em: http://www.inmet. gov.br/portal/index.php?r=bdmep/bdmep

Karaseva MO, Prakash S, Gairola RM. Validation of high-resolution TRMM-3B43 precipitation product using rain gauge measurements over Kyrgyzstan. Theoretical and Applied Climatology 2012; 108(1-2): 147-157. http:// dx.doi.org/10.1007/s00704-011-0509-6.

Kidder SQ, Haar THV. Satellite meteorology: an Introduction. San Diego: Academic Press; 1995.

Kummerow C, Simpson J, Thiele O, Barnes W, Chang ATC, Stocker E et al. The status of the Tropical Rainfall Measuring Mission (TRMM) after Two Years in orbit. Journal of Applied Meteorology 2000; 39(12): 1965-1982. http://dx.doi.org/10.1175/1520-0450(2001)040<1965:TS OTTR $>2.0 . \mathrm{CO} ; 2$.

Longo M, Camargo R, Silva Dias MAF. Análise das características dinâmicas e sinóticas de um evento de friagem durante a estação chuvosa no sudoeste da Amazônia. Revista Brasileira de Meteorologia 2004; 19(1): 59-72.

Marengo JA, Betts R, coordenadores. Riscos das mudanças climáticas no Brasil. Análise conjunta Brasil-Reino Unido sobre os impactos das mudanças climáticas e do desmatamento na Amazônia. São Paulo: CCST-INPE; 2011.

Marengo JA, Nobre CA, Tomasella J, Cardoso MF, Oyama MD. Hydro-climate and ecological behaviour of the drought of Amazonia in 2005. Philosophical transactions of the
Royal Society 2008; 363(1498): 1773-1778. http://dx.doi. org/10.1098/rstb.2007.0015. PMid:18270160.

Molion LCB. Climatologia dinâmica da Região Amazônica: mecanismos de precipitação. Revista Brasileira de Meteorologia 1987; 2(12): 107-117.

Nobre CA, Obregón GO, Marengo JA, Fu R, Poveda G. Characteristics of Amazonian climate: main features. In: Keller M, Bustamante M, Gash J, Dias PS, editores. Amazonia and global change. Washington: American Geophysical Union; 2009. http://dx.doi.org/10.1029/2008GM000720.

Nóbrega RS, Souza EP, Galvíncio JD. Análise da estimativa de precipitação do TRMM na Sub-Bacia da Amazônia ocidental. Revista de Geografia (Recife) 2008, 25(1): 06-20.

Oliveira JF Jr, Delgado RC, Gois G, Lannes A, Dias FO, Souza JC et al. Análise da precipitação e sua relação com sistemas meteorológicos em Seropédica, Rio de Janeiro. Revista Floresta e Ambiente 2014; 21(2): 140-149. http:// dx.doi.org/10.4322/floram.2014.030.

R Development Core Team. R: a language and environment for statistical computing [online]. Vienna, AT: R Foundation for Statistical Computing. [citado 2014 jul. 22]. Disponível em: http://www.R-project.org/

Reboita MS, Gan MA, Rocha RP, Ambrizzi T. Regimes de precipitação na América do Sul: uma revisão bibliográfica. Revista Brasileira de Meteorologia 2010; 25(2): 185-204. http://dx.doi.org/10.1590/S0102-77862010000200004.

Tropical Rainfall Measuring Mission - TRMM. Mission overview [online]. [citado 2014 jul. 22]. Disponível em: http://trmm.gsfc.nasa.gov

Vila DA, Gonçalves LGG, Toll DL, Rozante JR. Statistical evaluation of combined daily gauge observations and rainfall satellite estimates over continental south America. Bulletin of the American Meteorological Society 2009; 10(2): 533-542.

Willmott CJ, Ackleson SG, Davis JJ, Feddema JJ, Klink KM, Legates DR et al. Statistics for the evaluation and comparison of models. Journal of Geography Research 1985; 90(5): 8995-9005. 